\title{
MEAN REVERSION FOR HJMM FORWARD RATE MODELS
}

\author{
ANNA RUSINEK,* Institute of Mathematics, Polish Academy of Sciences
}

\begin{abstract}
We examine the long-time behavior of forward rates in the framework of Heath-JarrowMorton-Musiela models with infinite-dimensional Lévy noise. We give an explicit condition under which the rates have a mean reversion property. In a special case we show that this condition is fulfilled for any Lévy process with variance smaller than a given constant, depending only on the state space and the volatility.
\end{abstract}

Keywords: Term structure of interest rates; stationary distribution; infinite-dimensional model

2010 Mathematics Subject Classification: Primary 91B70

Secondary 60G51; 60H15

\section{Introduction}

In this paper we investigate whether certain models of bond markets can reflect the following property observed in the market: rates drop when they are high and rise when they are low. This behavior is called mean reversion. Since the mean reversion property does not reveal itself over a short horizon, we examine the long-time behavior. Whenever a process converges in law as time tends to $\infty$, it also has a tendency to remain close, over a long horizon, to an average value.

We give a sufficient condition for the mean reversion in Heath-Jarrow-Morton-Musiela (HJMM) models with infinite-dimensional Lévy noise. This is an extension of the result of Tehranchi [13], who considered models with Wiener noise on a weighted Sobolev space. In order to present a volatility example given by a Nemycki operator, which is used in applications, we present an analogous result on a weighted $L^{2}$ space. We also look more closely at the Laplace exponent of the driving process $Z$. In the case of one-dimensional Lévy noise with no negative jumps we derive the following simple and meaningful sufficient condition for the mean reversion in $L^{2}$ :

$$
\mathrm{E}|Z(1)|^{2}<K
$$

where $K>0$ depends only on the weight function and the volatility, but does not depend on the Lévy process $Z$. We also present a new proof of the Lipschitz property of the HeathJarrow-Morton mapping. The obtained Lipschitz constant of the mapping is smaller than the one obtained by Filipović and Tappe [6] or Peszat and Zabczyk [10]. Since these authors considered only the existence of solutions, it does not matter how large the Lipschitz constant is as long as it is finite. However, it is of importance for the mean reversion: the smaller the Lipschitz constant, the weaker the condition to ensure mean reversion. The proofs of the results regarding the mean reversion property are based on the general theorem, Theorem A.1,

Received 23 June 2009; revision received 18 February 2010.

* Postal address: Burgemeester van Stamplein 178, 2132BH Hoofddorp, The Netherlands.

Email address: annarusinek@yahoo.ca 
in which we present a sufficient condition for the existence of a stationary distribution for a general stochastic evolution equation driven by an infinite-dimensional Lévy process. This is an extension of the result of Chojnowska-Michalik [3], who considered equations with constant coefficients (see also [4, p. 105] for the Wiener case). Invariant distributions for the HJMM models with constant coefficients are discussed in [7], [8], and [15].

In Section 2 we establish the notation and present the HJMM model of the forward rate. In Section 3 our main results are stated. Section 4 contains some lemmas needed for the proofs of the theorems. These proofs are then given in Section 5. Section 6 deals with mean reversion under the objective measure.

\section{The HJMM model}

We start with the background necessary to present the model and state our results.

Let $\left(U,\langle\cdot, \cdot\rangle_{U}\right),\left(H,\langle\cdot, \cdot\rangle_{H}\right)$ be two separable Hilbert spaces. By $L(U, H)$ we denote the space of linear continuous operators from $U$ into $H$. We abbreviate $L(U, \mathbb{R})$ to $U^{*}$ and $L(U, U)$ to $L(U)$.

Let $Z(t)_{t \geq 0}$ be a $U$-valued Lévy martingale, i.e. a process with independent and stationary increments with mean 0 . Its Laplace exponent $J: U \rightarrow(-\infty,+\infty]$ is then given by

$$
J(u)=\ln \mathrm{E}^{-\langle u, Z(1)\rangle_{U}},
$$

and its covariance operator $Q$ is given by

$$
Q u=\mathrm{E}\left[\langle u, Z(1)\rangle_{U} Z(1)\right], \quad u \in U .
$$

We will assume that $Q \in L(U)$.

Definition 2.1. For a Lévy process $Z$ with the Laplace exponent $J: U \rightarrow(-\infty,+\infty]$, we define functions $\lambda_{1}, \lambda_{2}, \lambda_{3}:[0,+\infty) \rightarrow[0,+\infty]$ by

$$
\begin{aligned}
& \lambda_{1}(r)=\sup _{\|u\|_{U} \leq r}\|D J(u)\|_{U^{*}}, \\
& \lambda_{2}(r)=\sup _{\|u\|_{U} \leq r}\left\|D^{2} J(u)\right\|_{L\left(U, U^{*}\right)}, \\
& \lambda_{3}(r)=\sup _{\|u\|_{U} \leq r}\left\|D^{3} J(u)\right\|_{L\left(U, L\left(U, U^{*}\right)\right)},
\end{aligned}
$$

where $D^{k} J$ denotes the $k$ th derivative of $J$ and $D^{1} J$ is abbreviated to $D J$.

Before we can present the HJMM model of the forward rates, we need to recall some facts and concepts from operator theory. First note that, for every $\varphi \in H^{*}$ and $T \in L(U, H)$, the mapping $\varphi \circ T$ belongs to $U^{*}$, so, from the Riesz theorem (see [16, p. 90]), it follows that there exists a unique element of $U$, which we will denote by $T^{*} \varphi$, such that, for every $u \in U$,

$$
\left\langle T^{*} \varphi, u\right\rangle_{U}=\varphi(T u)
$$

An operator $T \in L(U, H)$ is said to belong to the space of Hilbert-Schmidt operators, denoted by $\mathcal{L}^{2}(U, H)$, if

$$
\|T\|_{\mathcal{L}^{2}(U, H)}^{2}=\sum_{i=1}^{\infty}\left\|T e_{i}\right\|_{H}^{2}<+\infty,
$$


where $\left\{e_{i}\right\}_{i}$ is an orthonormal basis in $U$. For $\varphi \in H^{*}$ and $T \in \mathcal{L}^{2}(U, H)$, we have

$$
\left\|T^{*} \varphi\right\|_{U}=\|\varphi \circ T\|_{U^{*}} \leq\|\varphi\|_{H^{*}}\|T\|_{L(U, H)} \leq\|\varphi\|_{H^{*}}\|T\|_{\mathscr{L}^{2}(U, H)} .
$$

We will denote by $L^{1}$ the space of all functions $f: \mathbb{R}_{+} \rightarrow \mathbb{R}$ such that

$$
\|f\|_{1}=\int_{0}^{\infty}|f(x)| \mathrm{d} x<\infty .
$$

Note that if $H$ is a separable Hilbert space of real functions defined on $[0,+\infty)$ such that

$$
\|f\|_{1} \leq C\|f\|_{H} \quad \text { for all } f \in H \text { and some } C>0,
$$

then, for every $\xi \geq 0$, the functional $I_{\xi}: H \rightarrow \mathbb{R}$ given by $I_{\xi} f=\int_{0}^{\xi} f(x) \mathrm{d} x$ is bounded and

$$
\sup _{\xi \geq 0}\left\|T^{*} I_{\xi}\right\|_{U} \leq C\|T\|_{\mathscr{L}^{2}(U, H)} \quad \text { for all } T \in \mathcal{L}^{2}(U, H) .
$$

We are now ready to present the model. Let $\mathscr{H}$ be a separable Hilbert space of real functions defined on $[0,+\infty)$. Let $\sigma$ be a mapping from $\mathscr{H}$ into $\mathcal{L}^{2}(U, H)$, where $H$ is a subspace of $\mathscr{H}$ such that (2.2) holds. Let $Z(t)_{t \geq 0}$ be a $U$-valued Lévy process. The HJMM model of the forward rates on $\mathscr{H}$ driven by $Z$ with volatility $\sigma$ is given by the following Musiela equation:

$$
f_{t}=S(t) f_{0}+\int_{0}^{t} S(t-s)\left(F_{\mathrm{HJM}} \circ \sigma\right)\left(f_{s}\right) \mathrm{d} s+\int_{0}^{t} S(t-s) \sigma\left(f_{s}\right) \mathrm{d} Z(s),
$$

where, for $T \in \mathcal{L}^{2}(U, H)$, the function $F_{\mathrm{HJM}}(T)$ is given by

$$
F_{\mathrm{HJM}}(T)(\xi)=\frac{\partial}{\partial \xi} J\left(T^{*} I_{\xi}\right),
$$

and $S(t)_{t \geq 0}$ is the semigroup of shift operators, i.e. $(S(t) f)(\xi)=f(\xi+t)$. It is worthwhile to rewrite the equation as

$$
\mathrm{d} f_{t}=\left(\mathbb{D} f_{t}+\left(F_{\mathrm{HJM}} \circ \sigma\right)\left(f_{t}\right)\right) \mathrm{d} t+\sigma\left(f_{t}\right) \mathrm{d} Z(t),
$$

where $\mathbb{D}$ denotes the generator of $S(t)_{t \geq 0}$, with $(\mathbb{D} f)(\xi)=\partial f(\xi) / \partial \xi$.

\section{Statements of results}

This section contains our main results. We present an already known theorem, and then we give an extension of it. Later we present analogous results on a state space which has useful properties and, finally, we consider models with one-dimensional noise.

To formulate our results, we use the following notation. For a positive increasing function $w: \mathbb{R}_{+} \rightarrow \mathbb{R}_{+}$, write

$$
C_{w}=\left\|w^{-1 / 3}\right\|_{1}^{3 / 2}, \quad \widetilde{C}_{w}=\left\|w^{-1}\right\|_{1}^{1 / 2}, \quad \alpha_{w}=\inf _{x \geq 0} \frac{w^{\prime}(x)}{w(x)},
$$

and let $G_{w}$ denote the Hilbert space of all functions $f: \mathbb{R}_{+} \rightarrow \mathbb{R}$ such that

$$
\int_{0}^{\infty}|f(x)|^{2} w(x) \mathrm{d} x<\infty
$$

with inner product

$$
\langle f, g\rangle_{G_{w}}=\int_{0}^{\infty} f(x) g(x) w(x) \mathrm{d} x
$$




\subsection{Results in Sobolev space}

Tehranchi [13] considered HJMM models on the Sobolev space $H_{w}$ (under the assumption that, for $w$, we have $\widetilde{C}_{w}<+\infty$ ), defined as the space of absolutely continuous functions $f: \mathbb{R}_{+} \rightarrow \mathbb{R}$ for which the weak derivative $f^{\prime}$ belongs to $G_{w}$, with inner product

$$
\langle f, g\rangle_{H_{w}}=f(\infty) g(\infty)+\left\langle f^{\prime}, g^{\prime}\right\rangle_{G_{w}}
$$

The existence of $f(\infty)=\lim _{x \rightarrow \infty} f(x)$ follows from the assumption that $\widetilde{C}_{w}<+\infty$ (see [13] for details). We distinguish the subspace $H_{w}^{0}=\left\{f \in H_{w}: f(\infty)=0\right\}$.

Theorem 3.1. ([13].) Assume that $Z$ in (2.3) is a standard Wiener process, and let $L, M \geq 0$ be such that, for every $f, g \in H_{w}$, we have

$$
\|\sigma(f)-\sigma(g)\|_{\mathscr{L}^{2}\left(U, H_{w}^{0}\right)} \leq L\|f-g\|_{H_{w}}, \quad\|\sigma(f)\|_{\mathscr{L}^{2}\left(U, H_{w}^{0}\right)} \leq M .
$$

If

$$
L^{2}+8 C_{w} M L<\alpha_{w}
$$

then, for every $c \in \mathbb{R}$, there exists a unique measure $\mu^{c}$ such that $\mu^{c}$ is the law limit of $\left(f_{t}\right)_{t \geq 0}$,

$$
\lim _{t \rightarrow \infty} \mathcal{L}\left(f_{t}\right)=\mu^{c}
$$

if $f_{0}(\infty)=c$, where $\left(f_{t}\right)_{t \geq 0}$ denotes the solution to (2.3).

Before we give an extension of the above theorem, we state our first existence result for models with infinite-dimensional Lévy noise.

Theorem 3.2. Suppose that $\sigma: H_{w} \rightarrow \mathcal{L}^{2}\left(U, H_{w}^{0}\right)$ is Lipschitz and bounded, i.e. there exist $L, M \geq 0$ such that, for every $f, g \in H_{w}$, we have

$$
\begin{gathered}
\|\sigma(f)-\sigma(g)\|_{\mathcal{L}^{2}\left(U, H_{w}^{0}\right)} \leq L\|f-g\|_{H_{w}}, \\
\|\sigma(f)\|_{\mathcal{L}^{2}\left(U, H_{w}^{0}\right)} \leq M .
\end{gathered}
$$

If $C_{w}<+\infty$ and $\lambda_{3}\left(C_{w} M\right)<+\infty$, then, for every $h \in H_{w}$, there exists a unique solution $\left(f_{t}\right)_{t \geq 0} \subset H_{w}$ to the Musiela equation (2.3) with $f_{0}=h$.

Given the existence of the solution, we now present a sufficient condition for the solution to have the mean reversion property. Note that, for the Wiener process, we have $Q u=u$, $\lambda_{1}(r)=r, \lambda_{2}(r) \equiv 1$, and $\lambda_{3}(r) \equiv 0$; thus, the following result is a generalization of Theorem 3.1.

Theorem 3.3. Let $L, M \geq 0$ be given by (3.1) and (3.2). Write $r_{w}=C_{w}$ M. If

$$
L^{2}\|Q\|_{L(U)}+2\left[\lambda_{1}\left(r_{w}\right)+3 \lambda_{2}\left(r_{w}\right) r_{w}+\lambda_{3}\left(r_{w}\right) r_{w}^{2}\right] L<\alpha_{w}
$$

then, for every $c \in \mathbb{R}$, there exists a unique measure $\mu^{c}$ such that $\lim _{t \rightarrow \infty} \mathcal{L}\left(f_{t}\right)=\mu^{c}$, if $f_{0}(\infty)=c$. 


\subsection{Results in the extended $L^{2}$ space}

We start with presenting a space of functions which can also be chosen as a state space for the HJMM model. The advantage of using this space lies in the fact that it allows us to present a volatility example given by a Nemycki operator, which is used in applications. Therefore, we propose to consider the HJMM model on a Hilbert space obtained by extending $G_{w}$ in such a way that it contains constant functions.

Definition 3.1. Let 1 stand for the function $\mathbf{1}(x)=1, x \geq 0$. If, for $c \in \mathbb{R}$, the set of all functions $f: \mathbb{R}_{+} \rightarrow \mathbb{R}$ for which $f-c \mathbf{1} \in G_{w}$ is denoted by $G_{w}^{c}$, then $\widehat{G}_{w}$ is defined by

$$
\widehat{G}_{w}=\bigcup_{c \in \mathbb{R}} G_{w}^{c} .
$$

The space $\widehat{G}_{w}$ is endowed with the following inner product: if $f \in G_{w}^{c} \subset \widehat{G}_{w}$ and $g \in G_{w}^{d} \subset$ $\widehat{G}_{w}$, then

$$
\langle f, g\rangle_{\widehat{G}_{w}}=c d+\langle f-c \mathbf{1}, g-d \mathbf{1}\rangle_{G_{w}} .
$$

Remark 3.1. Both spaces $H_{w}, \widehat{G}_{w} \simeq \mathbb{R} \times G_{w}$. The space $H_{w}$ treats the pair $(c, f)$ as a function $h(\xi)=c-\int_{\xi}^{\infty} f(x) \mathrm{d} x$, while $\widehat{G}_{w}$ treats the pair $(c, f)$ as a function $g(\xi)=c+f(\xi)$.

Remark 3.2. The concept of the so-called short rate, defined as $r_{t}=f_{t}(0)$, plays an important role in financial applications, such as discounting and present values, and in turn in proofs regarding the absence of arbitrage in the market where we show that the process of discounted bond prices

$$
\exp \left[-\int_{0}^{t} r_{v} \mathrm{~d} v\right] P(t, \theta),
$$

is a local martingale, where $P(t, \theta)$ denotes the price at time $t$ of a bond maturing at time $\theta>t$. If $f_{t} \in H_{w}$ then $f_{t}(0)$ is well defined, but $f_{t}(0)$ may not exist if $f_{t} \in \widehat{G}_{w}$. If there exists a strong solution to (2.4) then there exists a continuous version of $f_{t}$ and the short rate can be defined as

$$
r_{t}=\lim _{x \rightarrow 0} \frac{1}{x} \int_{0}^{x} f_{t}(\xi) \mathrm{d} \xi .
$$

If there is no noise in (2.3), i.e. $f_{t}=S(t) f_{0}$, then $f_{t}(0)=f_{0}(t)$ and the short rate given by $r_{t}=f_{0}(t)$ is well defined for almost every $t \geq 0$ and $\int_{0}^{t} r_{v} \mathrm{~d} v$ is well defined for every $t \geq 0$. Whether this approach works for the general equation (2.3) is the subject of ongoing research (see also [7]).

Analogously to the analysis of the stochastic differential equation for $H_{w}$, we now present an existence result as well as a sufficient condition for the mean reversion property of the stochastic differential equation for $\widehat{G}_{w}$.

Theorem 3.4. Suppose that $\sigma: \widehat{G}_{w} \rightarrow \mathcal{L}^{2}\left(U, G_{w}\right)$ is Lipschitz and bounded, i.e. there exist $L, M \geq 0$ such that, for every $f, g \in \widehat{G}_{w}$, we have

$$
\begin{gathered}
\|\sigma(f)-\sigma(g)\|_{\mathscr{L}^{2}\left(U, G_{w}\right)} \leq L\|f-g\|_{\widehat{G}_{w}}, \\
\|\sigma(f)\|_{\mathscr{L}^{2}\left(U, G_{w}\right)} \leq M .
\end{gathered}
$$

If $\widetilde{C}_{w}<+\infty$ and $\lambda_{2}\left(\widetilde{C}_{w} M\right)<+\infty$, then, for every $h \in \widehat{G}_{w}$, there exists a unique solution $\left(f_{t}\right)_{t \geq 0} \subset \widehat{G}_{w}$ to the Musiela equation (2.3) with $f_{0}=h$. 
Theorem 3.5. Let $L, M \geq 0$ be given by (3.4) and (3.5). If

$$
L^{2}\|Q\|_{L(U)}+2\left[\lambda_{1}\left(\widetilde{C}_{w} M\right)+\lambda_{2}\left(\widetilde{C}_{w} M\right) \widetilde{C}_{w} M\right] L<\alpha_{w}
$$

then, for every $c \in \mathbb{R}$, there exists a unique measure $\mu^{c}$ such that $\lim _{t \rightarrow \infty} \mathcal{L}\left(f_{t}\right)=\mu^{c}$, if $f_{0} \in G_{w}^{c}$.

\subsection{Models with one factor}

In this section we look more closely at HJMM models with one-dimensional noise. We start with the result regarding HJMM models on $H_{w}$.

Theorem 3.6. Consider an HJMM model on $H_{w}$, driven by a Lévy martingale taking values in $\mathbb{R}$ with $\sigma: H_{w} \rightarrow H_{w}^{0}$ given by

$$
\sigma(f)(\xi)=\int_{\xi}^{\infty} \min \left\{\psi(x),\left|f^{\prime}(x)\right|\right\} \mathrm{d} x
$$

for some positive real function $\psi$. Assume that $r_{w}=C_{w}\|\psi\|_{G_{w}}<+\infty$. If $J^{(3)}(0)<+\infty$ and $J^{(3)}\left(r_{w}\right)<+\infty$, then, for every $h \in H_{w}$, there exists a unique solution $\left(f_{t}\right)_{t \geq 0} \subset H_{w}$ to the Musiela equation (2.3) with $f_{0}=h$. If, additionally,

$$
\begin{aligned}
\mathrm{E}|Z(1)|^{2}+2\left[J^{\prime}\left(r_{w}\right)+3 \max \left\{\mathrm{E}|Z(1)|^{2},\right.\right. & \left.J^{\prime \prime}\left(r_{w}\right)\right\} r_{w} \\
& \left.+\max \left\{\left|J^{(3)}(0)\right|,\left|J^{(3)}\left(r_{w}\right)\right|\right\} r_{w}{ }^{2}\right]<\alpha_{w}
\end{aligned}
$$

then, for every $c \in \mathbb{R}$, there exists a unique measure $\mu^{c}$ such that $\lim _{t \rightarrow \infty} \mathcal{L}\left(f_{t}\right) \rightarrow \mu^{c}$, if $f_{0}(\infty)=c$.

Example 3.1. Let $\sigma$ be given by (3.7), and let $Z(t)=N_{\lambda}(t)-\widetilde{N}_{\lambda}(t)$, where $N_{\lambda}$ and $\widetilde{N}_{\lambda}$ are two independent Poisson processes with intensity $\lambda$. We have

$$
\mathrm{E}|Z(1)|^{2}=2 \lambda, \quad J^{(3)}(u)=J^{\prime}(u)=\lambda\left(\mathrm{e}^{u}-\mathrm{e}^{-u}\right), \quad J^{\prime \prime}(u)=\lambda\left(\mathrm{e}^{u}+\mathrm{e}^{-u}\right) ;
$$

thus, condition (3.8) takes the form

$$
\mathrm{E}|Z(1)|^{2}<\alpha_{w}\left[1+\left(\mathrm{e}^{r_{w}}-\mathrm{e}^{-r_{w}}\right)\left(1+r_{w}^{2}\right)+3\left(\mathrm{e}^{r_{w}}+\mathrm{e}^{-r_{w}}\right) r_{w}\right]^{-1} .
$$

The remainder of this section will be devoted to HJMM models on $\widehat{G}_{w}$, with volatility given by a Nemycki operator.

Theorem 3.7. Consider an HJMM model on $\widehat{G}_{w}$, driven by a square-integrable Lévy martingale taking values in $\mathbb{R}$ with $\sigma: \widehat{G}_{w} \rightarrow G_{w}$ given by

$$
\sigma(f)(\xi)=\min \{\psi(\xi),|f(\xi)|\}
$$

for some positive $\psi \in G_{w}$. If $\widetilde{C}_{w}<+\infty$ and $J^{\prime \prime}\left(\|\psi\|_{1}\right)<+\infty$, then, for every $h \in \widehat{G}_{w}$, there exists a unique solution $\left(f_{t}\right)_{t \geq 0} \subset \widehat{G}_{w}$ to the Musiela equation (2.3) with $f_{0}=h$. If, additionally,

$$
\mathrm{E}|Z(1)|^{2}+2\left[J^{\prime}\left(\|\psi\|_{1}\right)+\max \left\{\mathrm{E}|Z(1)|^{2}, J^{\prime \prime}\left(\|\psi\|_{1}\right)\right\} \widetilde{C}_{w}\|\psi\|_{G_{w}}\right]<\alpha_{w}
$$

then, for every $c \in \mathbb{R}$, there exists a unique measure $\mu^{c}$ such that $\lim _{t \rightarrow \infty} \mathcal{L}\left(f_{t}\right) \rightarrow \mu^{c}$, if $f_{0} \in G_{w}^{c}$. 
Example 3.2. Let $\sigma$ be given by (3.9), and let $Z(t)=N_{\lambda}(t)-\widetilde{N}_{\lambda}(t)$, where $N_{\lambda}$ and $\widetilde{N}_{\lambda}$ are two independent Poisson processes with intensity $\lambda$. We have $\mathrm{E}|Z(1)|^{2}=2 \lambda, J^{\prime}(u)=\lambda\left(\mathrm{e}^{u}-\mathrm{e}^{-u}\right)$, and $J^{\prime \prime}(u)=\lambda\left(\mathrm{e}^{u}+\mathrm{e}^{-u}\right)$; thus, condition (3.10) takes the form

$$
\mathrm{E}|Z(1)|^{2}<\alpha_{w}\left[1+\mathrm{e}^{\|\psi\|_{1}}-\mathrm{e}^{-\|\psi\|_{1}}+\widetilde{C}_{w}\|\psi\|_{G_{w}}\left(\mathrm{e}^{\|\psi\|_{1}}+\mathrm{e}^{-\|\psi\|_{1}}\right)\right]^{-1} .
$$

In the next example we show that, for a given $w$ and $\sigma$, the solution has the mean reversion property for any Lévy process with no negative jumps and variance sufficiently small. We emphasize that the function $J$ does not appear in the condition presented in the example.

Example 3.3. Suppose that $w(x)=\mathrm{e}^{\alpha x}$ for some $\alpha>0$. Let $Z$ be a Lévy process with no negative jumps, and let $\sigma$ be given by (3.9). Then, for every initial condition, (2.3) has a unique solution and condition (3.10) is fulfilled if

$$
\mathrm{E}|Z(1)|^{2}<\alpha\left[1+2\|\psi\|_{1}+2 \alpha^{-1 / 2}\|\psi\|_{G_{w}}\right]^{-1} .
$$

We have $\alpha_{w}=\alpha$ and $\widetilde{C}_{w}=\alpha^{-1 / 2}$. Therefore, to see that (3.11) implies (3.10), it is enough to apply (4.1) and (4.6), below, and the Lagrange theorem, since

$$
J^{\prime}\left(\|\psi\|_{1}\right)=J^{\prime}\left(\|\psi\|_{1}\right)-J^{\prime}(0) \leq\|\psi\|_{1} \sup _{u \geq 0}\left|J^{\prime \prime}(u)\right| .
$$

\section{Lipschitz property of the HJM mapping}

First we show some facts about the function $J$ defined by (2.1). We see that

$$
\begin{aligned}
D J(u) p & =-\mathrm{e}^{-J(u)} \mathrm{E}\left[\mathrm{e}^{-\langle u, Z(1)\rangle_{U}}\langle p, Z(1)\rangle_{U}\right], \\
D J(0) p & =-\langle p, \mathrm{E} Z(1)\rangle_{U}, \\
\left(D^{2} J(u) p\right) q & =\mathrm{e}^{-J(u)} \mathrm{E}\left[\mathrm{e}^{-\langle u, Z(1)\rangle_{U}}\langle p, Z(1)\rangle_{U}\langle q, Z(1)\rangle_{U}\right]-D J(u) p D J(u) q, \\
\left(D^{2} J(0) p\right) q & =\langle Q p, q\rangle_{U} .
\end{aligned}
$$

It follows that

$$
\begin{aligned}
& \lambda_{1}(0)=0, \\
& \lambda_{2}(0)=\|Q\|_{L(U)} .
\end{aligned}
$$

Since $\left(D^{2} J(u) p\right) q=\left(D^{2} J(u) q\right) p$, we obtain

$$
\left(D^{2} J(u) p\right) p-\left(D^{2} J(u) q\right) q=\left(D^{2} J(u)(p+q)\right)(p-q) .
$$

Moreover, with the notation

$$
X=\mathrm{e}^{-\langle u, Z(1)\rangle_{U} / 2}, \quad Y=\mathrm{e}^{-\langle u, Z(1)\rangle_{U} / 2}\langle p, Z(1)\rangle_{U},
$$

we have $\left(D^{2} J(u) p\right) p=\mathrm{e}^{-2 J(u)}\left[\mathrm{E} X^{2} \mathrm{E} Y^{2}-(\mathrm{E} X Y)^{2}\right]$, so

$$
\left(D^{2} J(u) p\right) p \geq 0 \quad \text { for all } u, p \in U
$$

Now we will consider properties of the Laplace exponent of a one-dimensional Lévy process. 
Proposition 4.1. ([12, Proposition 2.1].) Let J be a Laplace exponent of a Lévy process taking values in $\mathbb{R}$. For every $k \geq 1$, we have

$$
\sup _{u \in[a, b]}\left|J^{(k)}(u)\right|=\max \left\{\left|J^{(k)}(a)\right|,\left|J^{(k)}(b)\right|\right\},
$$

where $J^{(k)}$ denotes the $k$ th derivative of $J$. If $Z$ does not have negative jumps then, for every $k \geq 2$, we have $\sup _{u \in[a, b]}\left|J^{(k)}(u)\right|=\left|J^{(k)}(a)\right|$, and if $Z$ does not have positive jumps then, for every $k \geq 2$, we have $\sup _{u \in[a, b]}\left|J^{(k)}(u)\right|=J^{(k)}(b)$.

Note that, for a one-dimensional Lévy process,

$$
\left|J^{\prime \prime}(0)\right|=\lambda_{2}(0)=\|Q\|_{L(U)}=\mathrm{E}|Z(1)|^{2} .
$$

Therefore, since $J^{\prime}(0)=0$ and $J^{\prime \prime}$ is always positive, as a corollary of the above proposition, we obtain

$$
\begin{gathered}
\sup _{u \in[0, b]}\left|J^{\prime}(u)\right|=J^{\prime}(b), \\
\sup _{u \in[0, b]}\left|J^{\prime \prime}(u)\right|=\max \left\{\mathrm{E}|Z(1)|^{2}, J^{\prime \prime}(b)\right\} .
\end{gathered}
$$

Furthermore, if $Z$ does not have negative jumps then

$$
\sup _{u \geq 0}\left|J^{\prime \prime}(u)\right|=\mathrm{E}|Z(1)|^{2} .
$$

The next lemma plays a crucial role in the proof of Theorem 3.2.

Lemma 4.1. Assume that $\|A\|_{\mathcal{L}^{2}\left(U, H_{w}^{0}\right)}$ and $\|B\|_{\mathcal{L}^{2}\left(U, H_{w}^{0}\right)} \leq M$, and let $r_{w}=C_{w} M$. Then

$$
\left\|F_{\mathrm{HJM}}(A)-F_{\mathrm{HJM}}(B)\right\|_{H_{w}^{0}} \leq\left(\lambda_{1}\left(r_{w}\right)+3 \lambda_{2}\left(r_{w}\right) r_{w}+\lambda_{3}\left(r_{w}\right) r_{w}{ }^{2}\right)\|A-B\|_{\mathcal{L}^{2}\left(U, H_{w}^{0}\right)} .
$$

Proof. From Hölder's inequality, we obtain $\left\|f^{\prime}\right\|_{1} \leq \widetilde{C}_{w}\|f\|_{H_{w}^{0}}$. Furthermore, since $f(\xi)=-\int_{\xi}^{\infty} f^{\prime}(x) \mathrm{d} x$, it follows that, for every $\xi \geq 0$, the functional $\delta_{\xi}: H_{w}^{0} \rightarrow \mathbb{R}$, given by $\delta_{\xi} f=f(\xi)$, is bounded. So if $T \in \mathcal{L}^{2}\left(U, H_{w}^{0}\right)$ then $\partial T^{*} I_{\xi} / \partial \xi=T^{*} \delta_{\xi}$, and

$$
\begin{aligned}
\frac{\partial}{\partial \xi} F_{\mathrm{HJM}}(T)(\xi) & =\frac{\partial}{\partial \xi} D J\left(T^{*} I_{\xi}\right)\left(T^{*} \delta_{\xi}\right) \\
& =D J\left(T^{*} I_{\xi}\right)\left(\frac{\partial}{\partial \xi} T^{*} \delta_{\xi}\right)+\left(D^{2} J\left(T^{*} I_{\xi}\right) T^{*} \delta_{\xi}\right) T^{*} \delta_{\xi} .
\end{aligned}
$$

With the notation $h=F_{\mathrm{HJM}}(A)-F_{\mathrm{HJM}}(B)$, we have

$$
\|h\|_{H_{w}^{0}}^{2}=\left\langle F_{\mathrm{HJM}}(A)-F_{\mathrm{HJM}}(B), h\right\rangle_{H_{w}^{0}}=I+I I+I I I+I V,
$$

where

$$
\begin{aligned}
I & =\int_{0}^{\infty} D J\left(A^{*} I_{\xi}\right)\left(\frac{\partial}{\partial \xi} A^{*} \delta_{\xi}-\frac{\partial}{\partial \xi} B^{*} \delta_{\xi}\right) h^{\prime}(\xi) w(\xi) \mathrm{d} \xi \\
I I & =\int_{0}^{\infty}\left(D J\left(A^{*} I_{\xi}\right)-D J\left(B^{*} I_{\xi}\right)\right) \frac{\partial}{\partial \xi} B^{*} \delta_{\xi} h^{\prime}(\xi) w(\xi) \mathrm{d} \xi \\
I I I & =\int_{0}^{\infty}\left(\left(D^{2} J\left(A^{*} I_{\xi}\right) A^{*} \delta_{\xi}\right) A^{*} \delta_{\xi}-\left(D^{2} J\left(A^{*} I_{\xi}\right) B^{*} \delta_{\xi}\right) B^{*} \delta_{\xi}\right) h^{\prime}(\xi) w(\xi) \mathrm{d} \xi \\
I V & =\int_{0}^{\infty}\left(\left(D^{2} J\left(A^{*} I_{\xi}\right)-D^{2} J\left(B^{*} I_{\xi}\right)\right) B^{*} \delta_{\xi}\right) B^{*} \delta_{\xi} h^{\prime}(\xi) w(\xi) \mathrm{d} \xi .
\end{aligned}
$$


Applying (4.2) with $u=A^{*} I_{\xi}, p=A^{*} \delta_{\xi}$, and $q=B^{*} \delta_{\xi}$, we conclude that

$$
I I I=\int_{0}^{\infty}\left(D^{2} J\left(A^{*} I_{\xi}\right)(A+B)^{*} \delta_{\xi}\right)(A-B)^{*} \delta_{\xi} h^{\prime}(\xi) w(\xi) \mathrm{d} \xi
$$

The following inequalities can be found in Filipović [5] (see inequality (3.7) and the proof of inequality (3.8) therein):

$$
\begin{gathered}
\|f\|_{1} \leq \bar{C}_{w}\|f\|_{H_{w}^{0}} \\
\left(\int_{0}^{\infty}\|u(\xi)\|_{U}^{4} w(\xi) \mathrm{d} \xi\right)^{1 / 2} \leq \widehat{C}_{w} \int_{0}^{\infty}\left\|\frac{\partial}{\partial \xi} u(\xi)\right\|_{U}^{2} w(\xi) \mathrm{d} \xi
\end{gathered}
$$

with $\bar{C}_{w} \leq C_{w}$ and $\widehat{C}_{w} \leq C_{w}$. Furthermore, since

$$
\begin{aligned}
\int_{0}^{\infty}\left\|\frac{\partial}{\partial \xi} T^{*} \delta_{\xi}\right\|_{U}^{2} w(\xi) \mathrm{d} \xi & =\int_{0}^{\infty} \sum_{i=1}^{\infty}\left|\left\langle\frac{\partial}{\partial \xi} T^{*} \delta_{\xi}, e_{i}\right\rangle_{U}\right|^{2} w(\xi) \mathrm{d} \xi \\
& =\sum_{i=1}^{\infty} \int_{0}^{\infty}\left|\frac{\partial}{\partial \xi}\left\langle T^{*} \delta_{\xi}, e_{i}\right\rangle_{U}\right|^{2} w(\xi) \mathrm{d} \xi \\
& =\sum_{i=1}^{\infty} \int_{0}^{\infty}\left|\frac{\partial}{\partial \xi}\left(T e_{i}\right)(\xi)\right|^{2} w(\xi) \mathrm{d} \xi
\end{aligned}
$$

we obtain

$$
\begin{aligned}
& \left(\int_{0}^{\infty}\left\|\frac{\partial}{\partial \xi} T^{*} \delta \xi\right\|_{U}^{2} w(\xi) \mathrm{d} \xi\right)^{1 / 2}=\|T\|_{\mathcal{L}^{2}\left(U, H_{w}^{0}\right)}, \\
& \left(\int_{0}^{\infty}\left\|T^{*} \delta_{\xi}\right\|_{U}^{4} w(\xi) \mathrm{d} \xi\right)^{1 / 4} \leq C_{w}{ }^{1 / 2}\|T\|_{\mathscr{L}^{2}\left(U, H_{w}^{0}\right)} .
\end{aligned}
$$

By (4.7) we obtain $\left\|A^{*} I_{\xi}\right\|_{U},\left\|B^{*} I_{\xi}\right\|_{U} \leq r_{w}$ for every $\xi \geq 0$, and, from the Lagrange theorem,

$$
\begin{gathered}
\left\|D J\left(A^{*} I_{\xi}\right)-D J\left(B^{*} I_{\xi}\right)\right\|_{U^{*}} \leq \lambda_{2}\left(r_{w}\right) C_{w}\|A-B\|_{\mathcal{L}^{2}\left(U, H_{w}^{0}\right)}, \\
\left\|D^{2} J\left(A^{*} I_{\xi}\right)-D^{2} J\left(B^{*} I_{\xi}\right)\right\|_{L\left(U, U^{*}\right)} \leq \lambda_{3}\left(r_{w}\right) C_{w}\|A-B\|_{\mathcal{L}^{2}\left(U, H_{w}^{0}\right)} .
\end{gathered}
$$

Therefore,

$$
\begin{aligned}
|I| & \leq \lambda_{1}\left(r_{w}\right) \int_{0}^{\infty}\left\|\frac{\partial}{\partial \xi}(A-B)^{*} \delta_{\xi}\right\|_{U}\left|h^{\prime}(\xi)\right| w(\xi) \mathrm{d} \xi \\
|I I| & \leq \lambda_{2}\left(r_{w}\right) C_{w}\|A-B\|_{\mathcal{L}^{2}\left(U, H_{w}^{0}\right)} \int_{0}^{\infty}\left\|\frac{\partial}{\partial \xi} B^{*} \delta_{\xi}\right\|_{U}\left|h^{\prime}(\xi)\right| w(\xi) \mathrm{d} \xi \\
|I I I| & \leq \lambda_{2}\left(r_{w}\right) \int_{0}^{\infty}\left\|(A+B)^{*} \delta_{\xi}\right\|_{U}\left\|(A-B)^{*} \delta_{\xi}\right\|_{U}\left|h^{\prime}(\xi)\right| w(\xi) \mathrm{d} \xi, \\
|I V| & \leq \lambda_{3}\left(r_{w}\right) C_{w}\|A-B\|_{\mathscr{L}^{2}\left(U, H_{w}^{0}\right)} \int_{0}^{\infty}\left\|B^{*} \delta_{\xi}\right\|_{U}^{2}\left|h^{\prime}(\xi)\right| w(\xi) \mathrm{d} \xi .
\end{aligned}
$$


Now, applying (4.9), (4.10), and Hölder's inequality, we obtain

$$
\begin{aligned}
|I| & \leq \lambda_{1}\left(r_{w}\right)\|A-B\|_{\mathcal{L}^{2}\left(U, H_{w}^{0}\right)}\|h\|_{H_{w}^{0}}, \\
|I I| & \leq \lambda_{2}\left(r_{w}\right) C_{w}\|A-B\|_{\mathcal{L}^{2}\left(U, H_{w}^{0}\right)}\|B\|_{\mathcal{L}^{2}\left(U, H_{w}^{0}\right)}\|h\|_{H_{w}^{0}} \\
& \leq \lambda_{2}\left(r_{w}\right) C_{w} M\|A-B\|_{\mathcal{L}^{2}\left(U, H_{w}^{0}\right)}\|h\|_{H_{w}^{0}}, \\
|I I I| & \leq \lambda_{2}\left(r_{w}\right) C_{w}\|A-B\|_{\mathcal{L}^{2}\left(U, H_{w}^{0}\right)}\|A+B\|_{\mathcal{L}^{2}\left(U, H_{w}^{0}\right)}\|h\|_{H_{w}^{0}} \\
& \leq 2 \lambda_{2}\left(r_{w}\right) C_{w} M\|A-B\|_{\mathcal{L}^{2}\left(U, H_{w}^{0}\right)}\|h\|_{H_{w}^{0}}, \\
|I V| & \leq \lambda_{3}\left(r_{w}\right) C_{w}{ }^{2}\|A-B\|_{\mathcal{L}^{2}\left(U, H_{w}^{0}\right)}\|B\|_{\mathcal{L}^{2}\left(U, H_{w}^{0}\right)}^{2}\|h\|_{H_{w}^{0}} \\
& \leq \lambda_{3}\left(r_{w}\right) C_{w}{ }^{2} M^{2}\|A-B\|_{\mathcal{L}^{2}\left(U, H_{w}^{0}\right)}\|h\|_{H_{w}^{0} .}
\end{aligned}
$$

Remark 4.1. For a weight function of the form $w(x)=\mathrm{e}^{\alpha x}$ with $\alpha>0$, we have $C_{w}=$ $3^{3 / 2} \alpha^{-3 / 2}$. Furthermore, (4.7) and (4.8) hold with $\bar{C}_{w}=2 \alpha^{-3 / 2}$ and $\widehat{C}_{w}=\alpha^{-3 / 2}$. Therefore, for $w(x)=\mathrm{e}^{\alpha x}$, we can obtain a smaller Lipschitz constant of $F_{\mathrm{HJM}}: \mathcal{L}^{2}\left(U, H_{w}^{0}\right) \rightarrow H_{w}^{0}$, namely

$$
L_{F_{\mathrm{HJM}}}=\lambda_{1}\left(\bar{r}_{\alpha}\right)+2 \lambda_{2}\left(\bar{r}_{\alpha}\right) \bar{r}_{\alpha}+\frac{1}{2} \lambda_{3}\left(\bar{r}_{\alpha}\right) \bar{r}_{\alpha}^{2},
$$

where $\bar{r}_{\alpha}=2 \alpha^{-3 / 2} M$.

The following lemma is used in the proof of Theorem 3.6.

Lemma 4.2. Assume that $f, g \in H_{w}^{0}$ are positive functions and that $\|f\|_{H_{w}^{0}},\|g\|_{H_{w}^{0}} \leq M$, and let $r_{w}=C_{w} M$. Then

$$
\left\|F_{\mathrm{HJM}}(f)-F_{\mathrm{HJM}}(g)\right\|_{H_{w}^{0}} \leq K\|f-g\|_{H_{w}^{0}},
$$

with

$$
K=J^{\prime}\left(r_{w}\right)+3 \max \left\{\mathrm{E}|Z(1)|^{2}, J^{\prime \prime}\left(r_{w}\right)\right\} r_{w}+\max \left\{\left|J^{(3)}(0)\right|,\left|J^{(3)}\left(r_{w}\right)\right|\right\} r_{w}{ }^{2}
$$

Proof. Clearly, for a positive function $f$, we have

$$
f^{*} I_{\xi}=\int_{0}^{\xi} f(x) \mathrm{d} x \in\left[0,\|f\|_{1}\right] \subset\left[0, C_{w}\|f\|_{H_{w}^{0}}\right],
$$

so $\lambda_{1}\left(r_{w}\right), \lambda_{2}\left(r_{w}\right)$, and $\lambda_{3}\left(r_{w}\right)$ in the proof of Lemma 4.1 can be replaced by $\sup _{u \in\left[0, r_{w}\right]}\left|J^{\prime}(u)\right|$, $\sup _{u \in\left[0, r_{w}\right]}\left|J^{\prime \prime}(u)\right|$, and $\sup _{u \in\left[0, r_{w}\right]}\left|J^{(3)}(u)\right|$, respectively, to which we apply (4.4), (4.5), and (4.3).

The following lemma is used in the proofs of Theorem 3.4 and Theorem 3.5.

Lemma 4.3. If $\|A\|_{\mathscr{L}^{2}\left(U, G_{w}\right)},\|B\|_{\mathscr{L}^{2}\left(U, G_{w}\right)} \leq M$ then

$$
\left\|F_{\mathrm{HJM}}(A)-F_{\mathrm{HJM}}(B)\right\|_{G_{w}} \leq\left(\lambda_{1}\left(\widetilde{C}_{w} M\right)+\lambda_{2}\left(\widetilde{C}_{w} M\right) \widetilde{C}_{w} M\right)\|A-B\|_{\mathcal{L}^{2}\left(U, G_{w}\right)} .
$$

Proof. With the notation $h=F_{\mathrm{HJM}}(A)-F_{\mathrm{HJM}}(B)$, we have

$$
\|h\|_{G_{w}}^{2}=\left\langle F_{\mathrm{HJM}}(A)-F_{\mathrm{HJM}}(B), h\right\rangle_{G_{w}}=I+I I,
$$


where

$$
\begin{aligned}
& I=\int_{0}^{\infty} D J\left(A^{*} I_{\xi}\right)\left(\frac{\partial}{\partial \xi} A^{*} I_{\xi}-\frac{\partial}{\partial \xi} B^{*} I_{\xi}\right) h(\xi) w(\xi) \mathrm{d} \xi \\
& I I=\int_{0}^{\infty}\left(D J\left(A^{*} I_{\xi}\right)-D J\left(B^{*} I_{\xi}\right)\right)\left(\frac{\partial}{\partial \xi} B^{*} I_{\xi}\right) h(\xi) w(\xi) \mathrm{d} \xi
\end{aligned}
$$

From Hölder's inequality, we have $\|f\|_{1} \leq \widetilde{C}_{w}\|f\|_{G_{w}}$; hence,

$$
\left\|T^{*} I_{\xi}\right\|_{U} \leq \widetilde{C}_{w}\|T\|_{\mathscr{L}^{2}\left(U, G_{w}\right)} .
$$

So $\left\|A^{*} I_{\xi}\right\|_{U},\left\|B^{*} I_{\xi}\right\|_{U} \leq \widetilde{C}_{w} M$ for every $\xi \geq 0$, and, from the Lagrange theorem,

$$
\left\|D J\left(A^{*} I_{\xi}\right)-D J\left(B^{*} I_{\xi}\right)\right\|_{U^{*}} \leq \lambda_{2}\left(\widetilde{C}_{w} M\right) \widetilde{C}_{w}\|A-B\|_{\mathcal{L}^{2}\left(U, G_{w}\right)} .
$$

Therefore,

$$
\begin{aligned}
& |I| \leq \lambda_{1}\left(\widetilde{C}_{w} M\right) \int_{0}^{\infty}\left\|\frac{\partial}{\partial \xi}(A-B)^{*} I_{\xi}\right\|_{U}|h(\xi)| w(\xi) \mathrm{d} \xi \\
& |I I| \leq \lambda_{2}\left(\widetilde{C}_{w} M\right) \widetilde{C}_{w}\|A-B\|_{\mathcal{L}^{2}\left(U, G_{w}\right)} \int_{0}^{\infty}\left\|\frac{\partial}{\partial \xi} B^{*} I_{\xi}\right\|_{U}|h(\xi)| w(\xi) \mathrm{d} \xi .
\end{aligned}
$$

We claim that if $T \in \mathcal{L}^{2}\left(U, G_{w}\right)$ then

$$
\left(\int_{0}^{\infty}\left\|\frac{\partial}{\partial \xi} T^{*} I_{\xi}\right\|_{U}^{2} w(\xi) \mathrm{d} \xi\right)^{1 / 2}=\|T\|_{\mathcal{L}^{2}\left(U, G_{w}\right)} .
$$

Indeed, we have

$$
\begin{aligned}
\int_{0}^{\infty}\left\|\frac{\partial}{\partial \xi} T^{*} I_{\xi}\right\|_{U}^{2} w(\xi) \mathrm{d} \xi & =\int_{0}^{\infty} \sum_{i=1}^{\infty} \mid\left\langle\frac{\partial}{\partial \xi} T^{*} I_{\xi},\left.\left.e_{i}\right|_{U}\right|^{2} w(\xi) \mathrm{d} \xi\right. \\
& =\sum_{i=1}^{\infty} \int_{0}^{\infty}\left|\frac{\partial}{\partial \xi}\left\langle T^{*} I_{\xi}, e_{i}\right\rangle_{U}\right|^{2} w(\xi) \mathrm{d} \xi \\
& =\sum_{i=1}^{\infty} \int_{0}^{\infty}\left|\frac{\partial}{\partial \xi} \int_{0}^{\xi}\left(T e_{i}\right)(x) \mathrm{d} x\right|^{2} w(\xi) \mathrm{d} \xi \\
& =\sum_{i=1}^{\infty} \int_{0}^{\infty}\left|\left(T e_{i}\right)(\xi)\right|^{2} w(\xi) \mathrm{d} \xi
\end{aligned}
$$

Applying (4.11) and Hölder's inequality, we obtain

$$
\begin{aligned}
& |I| \leq \lambda_{1}\left(\widetilde{C}_{w} M\right)\|A-B\|_{\mathscr{L}^{2}\left(U, G_{w}\right)}\|h\|_{G_{w}}, \\
& |I I| \leq \lambda_{2}\left(\widetilde{C}_{w} M\right) \widetilde{C}_{w}\|A-B\|_{\mathcal{L}^{2}\left(U, G_{w}\right)}\|B\|_{\mathcal{L}^{2}\left(U, G_{w}\right)}\|h\|_{G_{w}} .
\end{aligned}
$$

In the next lemma we consider the mapping $F_{\mathrm{HJM}}: G_{w} \rightarrow G_{w}$ connected to a onedimensional Lévy process.

Lemma 4.4. Assume that $f, g \in G_{w}$ are positive functions, and that $\|f\|_{G_{w}},\|g\|_{G_{w}} \leq M$ and $\|f\|_{1},\|g\|_{1} \leq R$. Then

$$
\left\|F_{\mathrm{HJM}}(f)-F_{\mathrm{HJM}}(g)\right\|_{G_{w}} \leq \widetilde{K}\|f-g\|_{G_{w}},
$$

with $\widetilde{K}=J^{\prime}(R)+\max \left\{\mathrm{E}|Z(1)|^{2}, J^{\prime \prime}(R)\right\} \widetilde{C}_{w} M$. 
Proof. Note that $f^{*} I_{\xi}=\int_{0}^{\xi} f(x) \mathrm{d} x \in[0, R]$ and $g^{*} I_{\xi}=\int_{0}^{\xi} g(x) \mathrm{d} x \in[0, R]$; therefore, $\lambda_{1}\left(\widetilde{C}_{w} M\right)$ and $\lambda_{2}\left(\widetilde{C}_{w} M\right)$ in the proof of Lemma 4.3 can be replaced by $\sup _{u \in[0, R]}\left|J^{\prime}(u)\right|$ and $\sup _{u \in[0, R]}\left|J^{\prime \prime}(u)\right|$, respectively. It remains to apply (4.4) and (4.5).

We end this section with a short study of the properties of the shift semigroup. We claim that

$$
\|S(t) f\|_{G_{w}} \leq \mathrm{e}^{-\alpha_{w} t / 2}\|f\|_{G_{w}} \text { for all } f \in G_{w} .
$$

Indeed, note that the function $\bar{w}(x)=\mathrm{e}^{-\alpha_{w} x} w(x)$ is nondecreasing, since

$$
\bar{w}^{\prime}(x)=\mathrm{e}^{-\alpha_{w} x} w(x)\left[\frac{w^{\prime}(x)}{w(x)}-\alpha_{w}\right] \geq 0 .
$$

Hence,

$$
\begin{aligned}
\|S(t) f\|_{G_{w}}^{2} & =\int_{0}^{\infty}|f(x+t)|^{2} w(x) \mathrm{d} x \\
& \leq \int_{0}^{\infty}|f(x+t)|^{2} \mathrm{e}^{-\alpha_{w} t} w(x+t) \mathrm{d} x \\
& =\mathrm{e}^{-\alpha_{w} t} \int_{t}^{\infty}|f(\xi)|^{2} w(\xi) \mathrm{d} \xi \\
& \leq \mathrm{e}^{-\alpha_{w} t}\|f\|_{G_{w}}^{2} .
\end{aligned}
$$

Since $(S(t) f)^{\prime}=S(t)\left(f^{\prime}\right)$, inequality (4.12) is equivalent to

$$
\|S(t) f\|_{H_{w}^{0}} \leq \mathrm{e}^{-\alpha_{w} t / 2}\|f\|_{H_{w}^{0}} \text { for all } f \in H_{w}^{0} .
$$

\section{Proofs}

This section contains the proofs of the theorems. First we prove the existence results, and then we proceed with the results regarding the mean reversion property.

Proof of Theorem 3.2. By Theorem 9.29 of [11], a unique solution exists if $F_{\mathrm{HJM}} \circ \sigma: H_{w} \rightarrow$ $H_{w}$ is a Lipschitz mapping. From Lemma 4.1,

$$
\left\|\left(F_{\mathrm{HJM}} \circ \sigma\right)(f)-\left(F_{\mathrm{HJM}} \circ \sigma\right)(g)\right\|_{H_{w}^{0}} \leq K\left(C_{w} M\right) L\|f-g\|_{H_{w}},
$$

where $K(r)=\lambda_{1}(r)+3 \lambda_{2}(r) r+\lambda_{3}(r) r^{2}$. Furthermore, it follows from the Lagrange theorem that

$$
K(r) \leq \lambda_{1}(0)+4 \lambda_{2}(0) r+5 \lambda_{3}(r) r^{2}=4\|Q\|_{L(U)} r+5 \lambda_{3}(r) r^{2} .
$$

Proof of Theorem 3.4. We mimic the proof of Theorem 3.2 with appropriate adjustments. By Theorem 9.29 of [11], a unique solution exists if $F_{\mathrm{HJM}} \circ \sigma: \widehat{G}_{w} \rightarrow \widehat{G}_{w}$ is a Lipschitz mapping. From Lemma 4.3,

$$
\left\|\left(F_{\mathrm{HJM}} \circ \sigma\right)(f)-\left(F_{\mathrm{HJM}} \circ \sigma\right)(g)\right\|_{G_{w}} \leq \widetilde{K}\left(\widetilde{C}_{w} M\right) L\|f-g\|_{\widehat{G}_{w}},
$$

where $\widetilde{K}(r)=\lambda_{1}(r)+\lambda_{2}(r) r$. Furthermore, it follows from the Lagrange theorem that

$$
\widetilde{K}(r) \leq \lambda_{1}(0)+2 \lambda_{2}(r) r=2 \lambda_{2}(r) r .
$$

Proofs of Theorem 3.3 and Theorem 3.5 will be based on Theorem A.1, which we formulate and prove in Appendix A. 
Proof of Theorem 3.3. For $h \in H_{w}$, let $c=h(\infty)$, so $h^{c}=h-c \mathbf{1} \in H_{w}^{0}$. Define the mapping $\sigma^{c}: H_{w}^{0} \rightarrow L\left(U, H_{w}^{0}\right)$ by $\sigma^{c}(f)=\sigma(f+c \mathbf{1})$ and the mapping $F^{c}: H_{w}^{0} \rightarrow H_{w}^{0}$ by $F^{c}=F_{\mathrm{HJM}} \circ \sigma^{c}$. Consider the following equation on $H_{w}^{0}$ :

$$
\begin{aligned}
\mathrm{d} g_{t} & =\left(\mathbb{D} g_{t}+F^{c}\left(g_{t}\right)\right) \mathrm{d} t+\sigma^{c}\left(g_{t}\right) \mathrm{d} Z(t), \\
g_{0} & =h^{c} .
\end{aligned}
$$

Let us apply condition (A.4) from Theorem A.1. We have $L_{\sigma^{c}}=L$, so, from Lemma 4.1,

$$
L_{F^{c}}=\left(\lambda_{1}\left(r_{w}\right)+3 \lambda_{2}\left(r_{w}\right) r_{w}+\lambda_{3}\left(r_{w}\right) r_{w}^{2}\right) L .
$$

Hence, condition (3.3) states that $2 L_{F^{c}}+L_{\sigma^{c}}{ }^{2}\|Q\|_{L(U)}<2 \omega_{\mathbb{D}}$, since from (4.13) we get $\omega_{\mathbb{D}}=\frac{1}{2} \alpha_{w}$. It follows that $\mathcal{L}\left(g_{t}\right)$ converges to $\mathcal{L}\left(\mathcal{X}_{c}\right)$ for a random variable $\mathcal{X}_{c}$ such that $\mathrm{E} \mathcal{X}_{c}{ }^{2}<+\infty$. If $\left(g_{t}\right)_{t \geq 0}$ is a solution to (5.1) then $\left(f_{t}\right)_{t \geq 0}$ given by $f_{t}=g_{t}+c \mathbf{1}$ is a solution to

$$
\mathrm{d} f_{t}=\left(\mathbb{D} f_{t}+\left(F_{\mathrm{HJM}} \circ \sigma\right)\left(f_{t}\right)\right) \mathrm{d} t+\sigma\left(f_{t}\right) \mathrm{d} Z(t), \quad f_{0}=h .
$$

So $\mathcal{L}\left(f_{t}\right)$ converges to $\mathcal{L}\left(\mathcal{X}_{c}+c \mathbf{1}\right)$.

Proof of Theorem 3.5. The proof follows similar lines to that of Theorem 3.3. For $h \in G_{w}^{c}$, let $h^{c}=h-c \mathbf{1} \in G_{w}$. Define $\sigma^{c}: G_{w} \rightarrow L\left(U, G_{w}\right)$ by $\sigma^{c}(f)=\sigma(f+c \mathbf{1})$ and $F^{c}: G_{w} \rightarrow$ $G_{w}$ by $F^{c}=F_{\mathrm{HJM}} \circ \sigma^{c}$. Consider the following equation on $G_{w}$ :

$$
\begin{aligned}
\mathrm{d} g_{t} & =\left(\mathbb{D} g_{t}+F^{c}\left(g_{t}\right)\right) \mathrm{d} t+\sigma^{c}\left(g_{t}\right) \mathrm{d} Z(t), \\
g_{0} & =h^{c} .
\end{aligned}
$$

We apply condition (A.4) from Theorem A.1. We have $L_{\sigma^{c}}=L$, so, from Lemma 4.3,

$$
L_{F^{c}}=\left(\lambda_{1}\left(\widetilde{C}_{w} M\right)+\lambda_{2}\left(\widetilde{C}_{w} M\right) \widetilde{C}_{w} M\right) L .
$$

From (4.12), we get $\omega_{\mathbb{D}}=\frac{1}{2} \alpha_{w}$; thus, condition (3.6) states that

$$
2 L_{F^{c}}+L_{\sigma^{c}}^{2}\|Q\|_{L(U)}<2 \omega_{\mathbb{D}} .
$$

As in the proof of Theorem 3.3, we conclude that $\mathcal{L}\left(g_{t}\right)$ converges to $\mathcal{L}\left(\mathcal{X}_{c}\right)$ for a random variable $\mathcal{X}_{c}$ such that $\mathrm{E} \mathcal{X}_{c}{ }^{2}<+\infty$, and if $\left(g_{t}\right)_{t \geq 0}$ is a solution to (5.2) then $\left(f_{t}\right)_{t \geq 0}$ given by $f_{t}=g_{t}+c \mathbf{1}$ is a solution to

$$
\mathrm{d} f_{t}=\left(\mathbb{D} f_{t}+\left(F_{\mathrm{HJM}} \circ \sigma\right)\left(f_{t}\right)\right) \mathrm{d} t+\sigma\left(f_{t}\right) \mathrm{d} Z(t), \quad f_{0}=h .
$$

So $\mathcal{L}\left(f_{t}\right)$ converges to $\mathcal{L}\left(\mathcal{X}_{c}+c \mathbf{1}\right)$.

Proof of Theorem 3.6. It is enough to follow the proof of Theorem 3.3, applying Lemma 4.2 with $M=\|\psi\|_{G_{w}}$, since, for $\sigma^{c}: H_{w}^{0} \rightarrow H_{w}^{0}$ given by

$$
\sigma^{c}(f)(\xi)=\int_{\xi}^{\infty} \min \left\{\psi(x),\left|f^{\prime}(x)\right|\right\} \mathrm{d} x,
$$

we have

$$
\left\|\sigma^{c}(f)-\sigma^{c}(g)\right\|_{H_{w}^{0}} \leq\|f-g\|_{H_{w}^{0}}, \quad\left\|\sigma^{c}(f)\right\|_{H_{w}^{0}} \leq\|\psi\|_{G_{w}} .
$$


Proof of Theorem 3.7. For the mapping $\sigma^{c}: G_{w} \rightarrow G_{w}$ given by

$$
\sigma^{c}(f)(\xi)=\min \{\psi(\xi),|f(\xi)+c|\},
$$

we have

$$
\left\|\sigma^{c}(f)-\sigma^{c}(g)\right\|_{G_{w}} \leq\|f-g\|_{G_{w}}, \quad\left\|\sigma^{c}(f)\right\|_{G_{w}} \leq\|\psi\|_{G_{w}}, \quad\left\|\sigma^{c}(f)\right\|_{1} \leq\|\psi\|_{1} .
$$

Therefore, it is enough to follow the proof of Theorem 3.5, applying Lemma 4.4 with $R=\|\psi\|_{1}$ and $M=\|\psi\|_{G_{w}}$.

Remark 5.1. The same proof works for all Nemycki-type volatilities of the form

$$
\sigma(f)(\xi)=v(\xi, f(\xi))
$$

where $v: \mathbb{R}_{+} \times \mathbb{R} \rightarrow \mathbb{R}$ satisfies

$$
|v(\xi, x)-v(\xi, y)| \leq|x-y|, \quad 0 \leq v(\xi, x) \leq \psi(\xi)
$$

\section{Mean reversion under the objective measure}

In this section we discuss the case in which the HJMM dynamics correspond to the objective measure and, therefore, the mean reverting behavior of interest rates has an economic meaning. We will touch upon only a few aspects of the theory (see [2, pp. 194-200] and [13], where models with infinite Wiener noise are considered). We will assume that the Gaussian part of $Z$ is a standard Wiener process $W$ with covariance operator Id. Let $v$ be the Lévy measure of $Z$, i.e. $v(\{0\})=0$, and, for a Borel subset of $U$ such that $\bar{\Gamma} \subset U \backslash\{0\}$, we have

$$
v(\Gamma)=\mathrm{E}\left[\sum_{0<t \leq 1} \mathbf{1}_{\Gamma}\left(Z(t)-Z\left(t^{-}\right)\right)\right],
$$

where $Z\left(t^{-}\right)=\lim _{s \rightarrow t^{-}} Z(s)$. By $J_{v}$ we will denote the Laplace exponent of the jump part of $Z$, i.e.

$$
J_{v}(u)=\int_{U}\left(\mathrm{e}^{-\langle u, z\rangle_{U}}-1+\langle u, z\rangle_{U}\right) v(\mathrm{~d} z),
$$

and $\lambda_{k}^{(v)}$ will denote the function $\lambda_{k}$ connected to $J_{v}$, i.e.

$$
\lambda_{k}^{(v)}(r)=\sup _{\|u\|_{U} \leq r}\left\|D^{k} J_{v}(u)\right\|_{X_{k}}
$$

where $X_{0}=\mathbb{R}$ and $X_{k}=L\left(U, X_{k-1}\right), k \geq 1$. As in [1], we consider the following dynamics of the forward rates on $H$ :

$$
f_{t}=S(t) f_{0}+\int_{0}^{t} S(t-s) \widetilde{a}_{s} \mathrm{~d} s+\int_{0}^{t} S(t-s) \widetilde{\sigma}_{s} \mathrm{~d} Z(t),
$$

where $f_{0} \in H$, the process $\left(\widetilde{a}_{t}\right)_{t \geq 0}$ takes values in $H$, and the process $\left(\widetilde{\sigma}_{t}\right)_{t \geq 0}$ takes values in $\mathcal{L}^{2}(U, H)$. If $Z$ in the above equation is a Lévy process with respect to a measure locally equivalent to the martingale measure (see [1, Proposition 5.6]), then there exist a predictable 
process $\left(\varphi_{t}\right)_{t \geq 0}$ with values in $U$ and a measurable positive function $(\psi(t, z))_{t \geq 0, z \in U}$ such that the process $\left(\widetilde{\widetilde{a}}_{t}\right)_{t \geq 0}$ satisfies the following relation (see [1, Equation (5.36)]):

$$
\begin{aligned}
\int_{0}^{\xi} \widetilde{a}_{t}(x) \mathrm{d} x= & \frac{1}{2} \sum_{i=1}^{\infty}\left(\int_{0}^{\xi}\left(\widetilde{\sigma}_{t} e_{i}\right)(x) \mathrm{d} x\right)^{2}-\int_{0}^{\xi}\left(\widetilde{\sigma}_{t} \varphi_{t}\right)(x) \mathrm{d} x+\int_{U} \int_{0}^{\xi}\left(\widetilde{\sigma}_{t} z\right)(x) \mathrm{d} x v(\mathrm{~d} z) \\
& +\int_{U}\left(\exp \left[-\int_{0}^{\xi}\left(\widetilde{\sigma}_{t} z\right)(x) \mathrm{d} x\right]-1\right) \psi(t, z) v(\mathrm{~d} z) .
\end{aligned}
$$

We will assume that there exist a mapping $\sigma$ from $H$ into $\mathcal{L}^{2}(U, H)$ and mappings $\phi_{1}$ and $\phi_{2}$ from $H$ into $U$ such that $\widetilde{\sigma}_{t}=\sigma\left(f_{t}\right), \varphi_{t}=\phi_{1}\left(f_{t}\right)$, and $\psi(t, z)=\left\langle\phi_{2}\left(f_{t}\right), z\right\rangle_{U}$ for every $z$ from the support of $\nu$. This is a rather restrictive assumption, since positivity of $\psi$ implies that if $z$ belongs to the support of $v$ then $-z$ does not. For an $\mathbb{R}$-valued Lévy process with no negative jumps however, $v$ satisfies this constraint. With the above assumptions about the coefficients, $f_{t}$ becomes a solution to the following equation on $H$ :

$$
f_{t}=S(t) f_{0}+\int_{0}^{t} S(t-s) a\left(f_{s}\right) \mathrm{d} s+\int_{0}^{t} S(t-s) \sigma\left(f_{s}\right) \mathrm{d} Z(t),
$$

where $a: H \rightarrow H$ is given by

$$
a(f)=F_{\mathrm{HJM}}^{W}(\sigma(f))-\sigma(f) \phi_{1}(f)+\sigma(f) \int_{U} z \nu(\mathrm{d} z)-\mathcal{T}(\sigma(f)) \phi_{2}(f) .
$$

Here $F_{\mathrm{HJM}}^{W}$ is the HJM mapping connected to the Wiener process $W$ and $\mathcal{T}$ denotes the following mapping from $\mathcal{L}^{2}(U, H)$ into $L(U, H)$ :

$$
(\mathcal{T}(A) p)(\xi)=\left(D^{2} J_{\nu}\left(A^{*} I_{\xi}\right)\left(\frac{\partial}{\partial \xi} A^{*} I_{\xi}\right)\right) p, \quad p \in U, \xi \geq 0 .
$$

Following the proof of Lemma 4.1 with $D J$ replaced by $D^{2} J_{v}$ we find that if $\|A\|_{\mathscr{L}^{2}\left(U, H_{w}^{0}\right)}$, $\|B\|_{\mathcal{L}^{2}\left(U, H_{w}^{0}\right)} \leq M$, and $r_{w}=C_{w} M$, then

$$
\begin{aligned}
\|\mathcal{T}(A) p-\mathcal{T}(B) q\|_{H_{w}^{0}} & \\
\leq & \left(\lambda_{2}^{(v)}\left(r_{w}\right)+\lambda_{3}^{(v)}\left(r_{w}\right) r_{w}\right) M\|p-q\|_{U} \\
& \quad+\left(\lambda_{2}^{(v)}\left(r_{w}\right)+3 \lambda_{3}^{(v)}\left(r_{w}\right) r_{w}+\lambda_{4}^{(v)}\left(r_{w}\right) r_{w}{ }^{2}\right)\|q\|_{U}\|A-B\|_{\mathcal{L}^{2}\left(U, H_{w}^{0}\right)} .
\end{aligned}
$$

Following the proof of Lemma 4.3 with $D J$ replaced by $D^{2} J_{v}$ we find that if $\|A\|_{\mathscr{L}^{2}\left(U, G_{w}\right)}$, $\|B\|_{\mathscr{L}^{2}\left(U, G_{w}\right)} \leq M$ then

$$
\begin{aligned}
\|\mathcal{T}(A) p-\mathcal{T}(B) q\|_{G_{w}} \leq & \lambda_{2}^{(v)}\left(\widetilde{C}_{w} M\right) M\|p-q\|_{U} \\
& +\left(\lambda_{2}^{(v)}\left(\widetilde{C}_{w} M\right)+\lambda_{3}^{(v)}\left(\widetilde{C}_{w} M\right) \widetilde{C}_{w} M\right)\|q\|_{U}\|A-B\|_{\mathcal{L}^{2}\left(U, G_{w}\right)} .
\end{aligned}
$$

To simplify the presentation, we restrict our attention to models on $H=H_{w}^{0}$ or $H=G_{w}$, and we assume that there exist $L, M \geq 0$ such that, for every $f, g \in H, i=1,2$, we have

$$
\begin{aligned}
\left\|\phi_{i}(f)-\phi_{i}(g)\right\|_{U} & \leq L\|f-g\|_{H}, & & \left\|\phi_{i}(f)\right\|_{U} \leq M, \\
\|\sigma(f)-\sigma(g)\|_{\mathcal{L}^{2}(U, H)} & \leq L\|f-g\|_{H}, & & \|\sigma(f)\|_{\mathcal{L}^{2}(U, H)} \leq M .
\end{aligned}
$$

Applying Theorem A.1 to (6.1) we obtain the following result. 
Proposition 6.1. Let

$$
\bar{K}_{v}=2 \lambda_{2}^{(v)}\left(r_{w}\right)+4 \lambda_{3}^{(v)}\left(r_{w}\right) r_{w}+\lambda_{4}^{(v)}\left(r_{w}\right) r_{w}^{2},
$$

where $r_{w}=C_{w} M$. If

$$
\|Q\|_{L(U)} L^{2}+8 r_{w} L+4 M L+2 L\left\|\int_{U} z v(\mathrm{~d} z)\right\|_{U}+2 \bar{K}_{v} M L<\alpha_{w}
$$

then there exists a unique stationary distribution for (6.1) on $H_{w}^{0}$. Let

$$
\widetilde{K}_{v}=2 \lambda_{2}^{(v)}\left(\widetilde{C}_{w} M\right)+\lambda_{3}^{(v)}\left(\widetilde{C}_{w} M\right) \widetilde{C}_{w} M
$$

If

$$
\|Q\|_{L(U)} L^{2}+4 \widetilde{C}_{w} M L+4 M L+2 L\left\|\int_{U} z v(\mathrm{~d} z)\right\|_{U}+2 \widetilde{K}_{v} M L<\alpha_{w}
$$

then there exists a unique stationary distribution for (6.1) on $G_{w}$.

\section{Appendix A}

We will consider processes on a complete probability space $(\Omega, \mathcal{F}, \mathrm{P})$. Let $\left(U,\|\cdot\|_{U}\right)$ and $\left(H,\|\cdot\|_{H}\right)$ be two separable Hilbert spaces, and let $Z(t)_{t \geq 0}$ be a Lévy martingale taking values in $U$ with covariance operator $Q \in L(U)$. We consider a stochastic equation on $H$ of the form

$$
\begin{aligned}
\mathrm{d} X & =(A X+F(X)) \mathrm{d} t+B(X) \mathrm{d} Z(t), \\
X(0) & =\eta,
\end{aligned}
$$

where $\eta \in H$, the linear operator $A$ has dense domain and in general may be unbounded, $F$ is a mapping from $H$ into $H$, and $B$ is a mapping from $H$ into $L(U, H)$.

The following theorem gives a sufficient condition for the above equation to have a stationary distribution.

Theorem A.1. Let $S_{A}(t)_{t \geq 0}$ be the semigroup generated by $A$, and let $\omega_{A}, L_{F}, L_{B}>0$ be such that

$$
\begin{gathered}
\left\|S_{A}(t) x\right\|_{H} \leq \mathrm{e}^{-\omega_{A} t}\|x\|_{H}, \\
\|F(x)-F(y)\|_{H} \leq L_{F}\|x-y\|_{H}, \\
\|B(x)-B(y)\|_{\mathcal{L}^{2}(U, H)} \leq L_{B}\|x-y\|_{H} .
\end{gathered}
$$

Let $\left(X_{t}^{\eta}\right)_{t \geq 0}$ denote the solution of $(A .1)$. If

$$
-2 \omega_{A}+2 L_{F}+L_{B}^{2}\|Q\|_{L(U)}<0
$$

then there exists a unique random variable $\mathcal{X} \in L^{2}(\Omega)$ such that, for every $\eta \in H$,

$$
\mathcal{L}\left(X_{t}^{\eta}\right) \rightarrow \mathcal{L}(\mathcal{X})
$$

Furthermore, if $Z$ is square integrable then condition (A.3) can be replaced by the following weaker condition:

$$
\|B(x)-B(y)\|_{L(U, H)} \leq K_{B}\|x-y\|_{H},
$$

and condition (A.4) can be replaced by

$$
-2 \omega_{A}+2 L_{F}+\mathrm{E}\|Z(1)\|_{U}^{2} K_{B}^{2}<0 .
$$


Remark A.1. Van Gaans [14] presented a similar result under a less restrictive condition for the semigroup. He assumed that only $\|S(t)\|_{L(H)} \leq C \mathrm{e}^{-\omega_{A} t}$ for some $C, \omega_{A}>0$. His sufficient condition for the existence of a stationary distribution is

$$
6 C^{2}\left(\frac{L_{F}^{2}}{\omega_{A}}+\mathrm{E}\|Z(1)\|_{U}^{2} K_{B}^{2}\right)<\omega_{A} .
$$

In the case in which $C=1$, the above condition is equivalent to

$$
\frac{L_{F}^{2}}{\omega_{A}}-\frac{\omega_{A}}{6}+\mathrm{E}\|Z(1)\|_{U}^{2} K_{B}^{2}<0 .
$$

This condition is stronger than ours since

$$
\frac{L_{F}^{2}}{\omega_{A}}-\frac{\omega_{A}}{6}+2 \omega_{A}-2 L_{F}=\frac{5 \omega_{A}^{2}+6\left(\omega_{A}-L_{F}\right)^{2}}{6 \omega_{A}}>0 .
$$

In order to prove Theorem A.1, we present the following extension of Theorem 6.3.2 of [4]. Theorem A.2. Assume that $F$ satisfies condition (A.2) and that there exists $C_{B}^{Q}>0$ such that

$$
\left\|B(x) Q^{1 / 2}-B(y) Q^{1 / 2}\right\|_{\mathcal{L}^{2}(U, H)} \leq C_{B}^{Q}\|x-y\|_{H} .
$$

Let $A_{n}=n A(n-A)^{-1}, n \in \mathbb{N}$, be the sequence of Yosida approximations of $A$, and let $\left(X_{t}^{\eta}\right)_{t \geq 0}$ denote the solution of (A.1). If there exist $N, \varepsilon>0$ such that, for every $x, y$ in $H$ and $n>N$,

$$
\begin{aligned}
& 2\left\langle A_{n}(x-y)+F(x)-F(y), x-y\right\rangle_{H}+\left\|B(x) Q^{1 / 2}-B(y) Q^{1 / 2}\right\|_{\mathcal{L}^{2}(U, H)}^{2} \\
& \quad \leq-\varepsilon\|x-y\|_{H}^{2}
\end{aligned}
$$

then there exists a unique random variable $\mathcal{X} \in L^{2}(\Omega)$ such that, for every $\eta \in H$,

$$
\mathcal{L}\left(X_{t}^{\eta}\right) \rightarrow \mathcal{L}(\mathcal{X})
$$

Before we prove Theorem A. 2 we define the double-sided Lévy process and formulate two lemmas.

For the process $Z(t)_{t \geq 0}$, let $\bar{Z}(t)_{t \in \mathbb{R}}$ denote the process defined by

$$
\bar{Z}(t)= \begin{cases}Z(t), & t \geq 0 \\ Z_{2}(-t), & t<0\end{cases}
$$

where $Z_{2}(t)_{t \geq 0}$ is a Lévy process with the same distribution as $Z(t)_{t \geq 0}$ and independent of $Z(t)_{t \geq 0}$.

Lemma A.1. ([11, Lemma D.3].) Let $\alpha(t)_{t \geq 0}$ be a predictable $H$-valued process, and let $\beta(t)_{t \geq 0}$ be a predictable $L(U, H)$-valued process such that

$$
\mathrm{E} \int_{t_{0}}^{T}\left(\|\alpha(t)\|_{H}^{2}+\left\|\beta(t) Q^{1 / 2}\right\|_{\mathcal{L}^{2}(U, H)}^{2}\right) \mathrm{d} t<\infty \text { for all } T>t_{0} .
$$

Then, for any $h \in H$, the process

$$
Y(t)=h+\int_{t_{0}}^{t} \alpha(s) \mathrm{d} s+\int_{t_{0}}^{t} \beta(s) \mathrm{d} \bar{Z}(s)
$$


is square integrable and

$$
\frac{\mathrm{d}}{\mathrm{d} t} \mathrm{E}\|Y(t)\|_{H}^{2}=\mathrm{E}\left[2\langle Y(t), \alpha(t)\rangle_{H}+\left\|\beta(t) Q^{1 / 2}\right\|_{\mathcal{L}^{2}(U, H)}^{2}\right] .
$$

Lemma A.2. Condition (A.6) implies that, for some $C_{1}>0$,

$$
2\left\langle A_{n} x+F(x), x\right\rangle_{H}+\left\|B(x) Q^{1 / 2}\right\|_{\mathcal{L}^{2}(U, H)}^{2} \leq-\frac{\varepsilon}{2}\|x\|_{H}^{2}+C_{1}
$$

for every $x$ in $H$ and $n>N$.

Proof. Letting $y=0$ in (A.6), we obtain

$$
I \leq-\varepsilon\|x\|_{H}^{2}+2\langle F(0), x\rangle_{H}+I I,
$$

where

$$
I=2\left\langle A_{n} x+F(x), x\right\rangle_{H}+\left\|B(x) Q^{1 / 2}\right\|_{\mathscr{L}^{2}(U, H)}^{2}
$$

and

$$
\begin{aligned}
I I= & \left\|B(x) Q^{1 / 2}\right\|_{\mathcal{L}^{2}(U, H)}^{2}-\left\|B(x) Q^{1 / 2}-B(0) Q^{1 / 2}\right\|_{\mathscr{L}^{2}(U, H)}^{2} \\
\leq & \left\|B(x) Q^{1 / 2}\right\|_{\mathcal{L}^{2}(U, H)}^{2}-\left(\left\|B(x) Q^{1 / 2}\right\|_{\mathscr{L}^{2}(U, H)}-\left\|B(0) Q^{1 / 2}\right\|_{\mathcal{L}^{2}(U, H)}\right)^{2} \\
= & \left\|B(0) Q^{1 / 2}\right\|_{\mathscr{L}^{2}(U, H)}^{2} \\
& +2\left\|B(0) Q^{1 / 2}\right\|_{\mathcal{L}^{2}(U, H)}\left(\left\|B(x) Q^{1 / 2}\right\|_{\mathcal{L}^{2}(U, H)}-\left\|B(0) Q^{1 / 2}\right\|_{\mathcal{L}^{2}(U, H)}\right) \\
\leq & \left\|B(0) Q^{1 / 2}\right\|_{\mathcal{L}^{2}(U, H)}^{2}+2\left\|B(0) Q^{1 / 2}\right\|_{\mathscr{L}^{2}(U, H)} C_{B}^{Q}\|x\|_{H} .
\end{aligned}
$$

Therefore,

$$
I \leq-\frac{\varepsilon}{2}\|x\|_{H}^{2}+a\|x\|_{H}^{2}+b\|x\|_{H}+c
$$

where

$$
\begin{aligned}
a & =-\frac{\varepsilon}{2}<0, \\
b & =2\left(\|F(0)\|_{H}+\left\|B(0) Q^{1 / 2}\right\|_{\mathscr{L}^{2}(U, H)} C_{B}^{Q}\right), \\
c & =\left\|B(0) Q^{1 / 2}\right\|_{\mathscr{L}^{2}(U, H)}^{2} .
\end{aligned}
$$

Hence,

$$
I \leq-\frac{\varepsilon}{2}\|x\|_{H}^{2}+C_{1}, \quad \text { with } \quad C_{1}=-\frac{b^{2}-4 a c}{4 a} .
$$

Proof of Theorem A.2. Let $\bar{Z}$ be defined by (A.7), and let $A_{n}=n A(n-A)^{-1}, n \in \mathbb{N}$, be the sequence of Yosida approximations of $A$. Denote by $X_{n}(t, s, \eta)$ the solution to the equation

$$
\mathrm{d} X_{n}=\left(A_{n} X_{n}+F\left(X_{n}\right)\right) \mathrm{d} t+B\left(X_{n}\right) \mathrm{d} \bar{Z}(t), \quad X_{n}(s)=\eta,
$$

and by $X(t, s, \eta)$ the solution to the equation

$$
\mathrm{d} X=(A X+F(X)) \mathrm{d} t+B(X) \mathrm{d} \bar{Z}(t), \quad X(s)=\eta .
$$

Then $X_{n}(t, s, \eta)$ converges in $L^{2}(\Omega)$ to $X(t, s, \eta)$. Fix $\eta \in H$ and $s \in \mathbb{R}$. Applying Lemma A.1 with

$$
Y(t)=X_{n}(t, s, \eta), \quad \alpha(t)=A_{n} X_{n}(t, s, \eta)+F\left(X_{n}(t, s, \eta)\right), \quad \beta(t)=B\left(X_{n}(t, s, \eta)\right),
$$


and Lemma A.2, we obtain

$$
\frac{\mathrm{d}}{\mathrm{d} t} \mathrm{E}\|Y(t)\|_{H}^{2} \leq-\frac{\varepsilon}{2} \mathrm{E}\|Y(t)\|_{H}^{2}+C_{1} .
$$

By Gronwall's lemma for every $t \geq s$, we have $\mathrm{E}\|Y(t)\|_{H}^{2} \leq 2 C_{1} \varepsilon^{-1}+\mathrm{E}\|Y(s)\|_{H}^{2}$. Since $X_{n}(t, s, \eta)$ converges in $L^{2}(\Omega)$ to $X(t, s, \eta)$, we also have

$$
\mathrm{E}\|X(t, s, \eta)\|_{H}^{2} \leq 2 C_{1} \varepsilon^{-1}+\|\eta\|_{H}^{2} \quad \text { for all } \eta \in H \text { and } t \geq s .
$$

Once the boundedness of the solutions has been obtained, we prove the existence of a unique stationary distribution. The first step will be to prove that there exists a law limit of $(X(\gamma, 0, \eta))_{\gamma}$ for some $\eta$ (to specify, we take $\eta=0$ ), and the second step will be to prove that, for any $\eta$, the law limit of $(X(\gamma, 0, \eta))_{\gamma}$ is the same.

Fix $\eta \in H$ and $\delta \geq \gamma>0$, and let $U_{n}(t)=X_{n}(t,-\gamma, 0)$ and $V_{n}(t)=X_{n}(t,-\delta, \eta)$. Then

$$
\mathrm{d}\left(U_{n}-V_{n}\right)=\left(A\left(U_{n}-V_{n}\right)+F\left(U_{n}\right)-F\left(V_{n}\right)\right) \mathrm{d} t+\left(B\left(U_{n}\right)-B\left(V_{n}\right)\right) \mathrm{d} \bar{Z}(t) .
$$

Applying Lemma A.1 with

$$
\begin{aligned}
& \widetilde{Y}(t)=U_{n}(t)-V_{n}(t), \\
& \widetilde{\alpha}(t)=A_{n}\left(U_{n}(t)-V_{n}(t)\right)+F\left(U_{n}(t)\right)-F\left(V_{n}(t)\right), \\
& \widetilde{\beta}(t)=B\left(U_{n}(t)\right)-B\left(V_{n}(t)\right),
\end{aligned}
$$

and condition (A.6), we conclude that

$$
\frac{\mathrm{d}}{\mathrm{d} t} \mathrm{E}\|\tilde{Y}(t)\|_{H}^{2} \leq-\varepsilon \mathrm{E}\|\tilde{Y}(t)\|_{H}^{2} .
$$

By Gronwall's lemma, for every $s \in \mathbb{R}$ and every $t \geq s$,

$$
\mathrm{E}\|\tilde{Y}(t)\|_{H}^{2} \leq \mathrm{e}^{-\varepsilon(t-s)} \mathrm{E}\|\tilde{Y}(s)\|_{H}^{2} .
$$

Letting $t=0$ and $s=-\gamma$, we obtain

$$
\mathrm{E}\left\|X_{n}(0,-\gamma, 0)-X_{n}(0,-\delta, \eta)\right\|_{H}^{2} \leq \mathrm{e}^{-\varepsilon \gamma} \mathrm{E}\left\|X_{n}(-\gamma,-\delta, \eta)\right\|_{H}^{2} .
$$

Since $X_{n}(t, s, \eta)$ converges in $L^{2}(\Omega)$ to $X(t, s, \eta)$,

$$
\mathrm{E}\|X(0,-\gamma, 0)-X(0,-\delta, \eta)\|_{H}^{2} \leq \mathrm{e}^{-\varepsilon \gamma} \mathrm{E}\|X(-\gamma,-\delta, \eta)\|_{H}^{2} .
$$

Letting $\eta=0$, we obtain

$$
\mathrm{E}\|X(0,-\gamma, 0)-X(0,-\delta, 0)\|_{H}^{2} \leq \mathrm{e}^{-\varepsilon \gamma} \mathrm{E}\|X(-\gamma,-\delta, 0)\|_{H}^{2} .
$$

Now, recalling (A.8),

$$
\mathrm{E}\|X(0,-\gamma, 0)-X(0,-\delta, 0)\|_{H}^{2} \leq \mathrm{e}^{-\varepsilon \gamma} 2 C_{1} \varepsilon^{-1} .
$$

It follows that $(X(0,-\gamma, 0))_{\gamma}$ is a Cauchy sequence in $L^{2}(\Omega)$, so there exists a random variable $X \in L^{2}(\Omega)$ such that $X(0,-\gamma, 0)$ converges to $X$ in $L^{2}(\Omega)$, which implies that $X(0,-\gamma, 0)$ converges to $\mathcal{X}$ also in law. Since $\mathcal{L}(X(0,-\gamma, 0))=\mathcal{L}(X(\gamma, 0,0))$, we also have

$$
\mathcal{L}(X(\gamma, 0,0)) \rightarrow \mathcal{L}(X) \quad \text { as } \gamma \rightarrow \infty
$$


To prove uniqueness of the stationary distribution, let $\delta=\gamma$ in (A.9). We obtain

$$
\mathrm{E}\|X(0,-\gamma, 0)-X(0,-\gamma, \eta)\|_{H}^{2} \leq \mathrm{e}^{-\varepsilon \gamma}\|\eta\|_{H}^{2} .
$$

So the $L^{2}(\Omega)$ limit, and then also the law limit, of $(X(0,-\gamma, \eta))_{\gamma}$ is $\mathcal{X}$ as well. Furthermore, since $\mathcal{L}(X(0,-\gamma, \eta))=\mathscr{L}(X(\gamma, 0, \eta))$, we also have

$$
\mathcal{L}(X(\gamma, 0, \eta)) \rightarrow \mathcal{L}(\mathcal{X}) \text { as } \gamma \rightarrow \infty
$$

Proof of Theorem A.1. It is sufficient to prove that condition (A.4), as well as condition (A.5), implies condition (A.6). This idea is due to Tehranchi [13]. First note that

$$
\left\|Q^{1 / 2}\right\|_{\mathcal{L}^{2}(U, H)}^{2}=\mathrm{E}\|Z(1)\|_{U}^{2}
$$

hence,

$$
\left(C_{B}^{Q}\right)^{2} \leq \min \left\{L_{B}^{2}\|Q\|_{L(U)}, \mathrm{E}\|Z(1)\|_{U}^{2} K_{B}{ }^{2}\right\} .
$$

Therefore, if (A.4) or (A.5) holds, then there exist $\varepsilon, N>0$ such that, for $n \geq N$, we have

$$
-\frac{2 \omega_{A} n}{n+\omega_{A}}+2 L_{F}+\left(C_{B}^{Q}\right)^{2}<-\varepsilon
$$

since $\omega_{A} n /\left(n+\omega_{A}\right) \rightarrow \omega_{A}$ as $n \rightarrow \infty$. For the Yosida approximations, $A_{n}$, we have

$$
\left\langle A_{n} h, h\right\rangle_{H} \leq-\frac{\omega_{A} n}{n+\omega_{A}}\|h\|_{H}^{2},
$$

since $\left\|S_{A}(t)\right\|_{L(H)} \leq \mathrm{e}^{-\omega_{A} t}$ (see the proof of Theorem 7 in [13]).

\section{Acknowledgements}

The author is greatly indebted to Professor Jerzy Zabczyk for drawing the author's attention to the $L^{2}$ space as a state space and for many stimulating conversations.

The author acknowledges the support of the EC FP6 MC-ToK programme SPADE2, MTKDCT-2004-014508 and the support of the Polish Ministry of Science and Education project 1PO 3A 034 29, Stochastic Evolution Equations with Lévy noise.

\section{References}

[1] BuöRK, T., Di Masi, G., Kabanov, Y. And RungGaldier, W. (1997). Towards a general theory of bond markets. Finance Stoch. 1, 141-174.

[2] Carmona, R. and Tehranchi, M. R. (2006). Interest Rate Models: An Infinite Dimensional Stochastic Analysis Perspective. Springer, Berlin.

[3] Chojnowska-Michalik, A. (1987). On processes of Ornstein Uhlenbeck type in Hilbert space. Stochastics 21, 251-286.

[4] Da Prato, G. and ZabczyK, J. (1996). Ergodicity for Infinite-Dimensional Systems. Cambridge University Press.

[5] Filipović, D. (2000). Consistency problems for HJM interest rate models. Doctoral Thesis, ETH Zürich.

[6] Filipović, D. ANd TAPPE, S. (2008). Existence of Lévy term structure models. Finance Stoch. 12, 83-115.

[7] Goldys, B. and Musiela, M. (1996). On partial differential equations related to term structure models. Preprint.

[8] MarinelLi, C. (2010). Well-posedness and invariant measures for HJM models with deterministic volatility and Lévy noise. Quant. Finance 10, 39-47.

[9] Musiela, M. (1993). Stochastic PDEs and term structure models. In Journées Internationales de Finance IGR-AFFI (La Baule, June 1993). 
[10] Peszat, S. and ZabczyK, J. (2007). Heath-Jarrow-Morton-Musiela equation of bond market. Preprint 677. Available at http://www.impan.gov.p1/Preprints/p677.pdf.

[11] Peszat, S. and Zabczyk, J. (2007). Stochastic Partial Differential Equations with Lévy Noise. Cambridge University Press.

[12] Rusinek, A. (2006). Invariant measures for forward rate HJM model with Lévy noise. Preprint 669. Available at http://www.impan.gov.pl/Preprints/p669.pdf.

[13] Tehranchi, M. (2005). A note on invariant measures for HJM models. Finance Stoch. 9, 389-398.

[14] VAN GaAns, O. (2005). Invariant measures for stochastic evolution equations with Hilbert space valued Lévy noise. Tech. Rep., Friedrich Schiller University. Available at http://www.math.leidenuniv.nl/ vangaans/ gaansrep1.pdf.

[15] VARgiolu, T. (1999). Invariant measures for the Musiela equation with deterministic diffusion term. Finance Stoch. 3, 483-492.

[16] Yosida, K. (1980). Functional Analysis, 6th edn. Springer, Berlin. 